\title{
RF Energy Harvesting with Multiple Sources in Wireless Mesh Network
}

\author{
K.N. Puniran ${ }^{\text {, Ahmad Robiah*2, Rudzidatul Akmam Dziyauddin }}{ }^{3}$ \\ ${ }^{1}$ Department Petrofac Engineering Services (Malaysia) Sdn Bhd \\ Jalan Ampang, Kuala Lumpur, Malaysia \\ ${ }^{2,3}$ Razak School of Engineering and Advanced Technology, Universiti Teknologi Malaysia \\ Jalan Semarak, Kuala Lumpur, Malaysia
}

\begin{tabular}{l}
\hline \hline Article Info \\
\hline Article history: \\
Received Nov 15, 2017 \\
Revised Jan 16, 2018 \\
Accepted Feb 21, 2018 \\
\hline
\end{tabular}

Keywords:

Co-channel Interference

Decode-and-forward

Energy Harvesting

Ergodic Capacity

Mesh Network

\begin{abstract}
Energy harvesting (EH) module for wireless sensor network has become a promising feature to prolong the conventional battery inside the devices. This emerging technology is gaining interest from sensor manufacturers as well as academicians across the globe. The concept of employing EH module must be cost effective and practical. In such, the use of EH module type besides $\mathrm{RF}$ is more realistic due to the size of the scavenger module, the availability of the resources and conversion efficiency. Most of the oil and gas plants have some drawbacks in scavenging RF from surrounding (i.e. router, Wi-Fi, base station, cell phone) due to its placement in remote area and thus limited energy sources could be a threat in this application. Multiple sources, including co-channel interference (CCI) in any constraint nodes is a feasible way of scavenging several wastes from ambient RF energy via wireless mesh topology. In this paper, a 3-node decode-and-forward (DF) model is proposed where the relay node is subject to an energy constraint. Multiple primary sources and CCI are added in the system model known as MultipleSource and Single-Relay (MSSR). A mathematical model is derived in Time Switching Relaying (TSR) and Power Splitting Relaying (PSR) schemes to obtain an average system throughput at a destination. Numerical simulation with respect to the average throughput and $\mathrm{EH}$ ratio was performed and compared with the Single-Source and Single-Relay (SSSR) and ideal receiver. By applying multiple sources and CCI as an energy enhancement at the constraint node, the optimal value of EH ratio for TSR can be reduced significantly by $10 \%$ as compared to the ideal receiver whereas the optimal value of EH ratio for PSR is outweigh TSR in terms of overall system throughput.
\end{abstract}

Copyright $@ 2018$ Institute of Advanced Engineering and Science. All rights reserved.

\section{Corresponding Author:}

Robiah Ahmad

Razak School of Engineering and Advanced Technology,

Universiti Teknologi Malaysia, Jalan Semarak, Kuala Lumpur, Malaysia

Email: robiahahmad@utm.my

\section{INTRODUCTION}

RF energy harvesting is a process to harness energy from ambient to provide power and store energy in wireless sensor devices. One of the key advantages of RF energy harvesting is the ability to increase overall system throughput at the destination. Apart from that, the overall cost on project implementation can be significantly reduced if wireless sensors are being deployed, hence will eliminate the needs of physical cable. Nonetheless, the return on investment is superior and outweigh the wired network. In terms of network efficiency, system latency can be drastically reduced since real-time data and sensor's scan rate can be set faster without losing the sensor power in lesser time, consequently increases power network range and improves performance [1]. 
Typically, wireless sensor operates in Industrial, Scientific and Medical (ISM) $2.4 \mathrm{GHz}$ radio band. The wireless sensor uses several techniques to coexist with other wireless applications in the same spectrum bands [2]. One of the techniques is the network segmentation where each segment has several wireless sensor devices appeared in the same physical space and the devices embed with a unique network identification (ID). The spectrum isolation is another coexistence technique to avoid interference between wireless applications operating in different portion of spectrum bands. In addition to that, wireless sensor devices are low-power devices, and this may aid to prevent from interfering with high-power applications. Another important key aspect of coexistence technique is the ability of the wireless sensor network in spatial diversity. It means the self-organizing mesh network can hop on different and several paths within its segment that may expose to different RF condition. The wireless sensor device can perform channel hopping and uses 15 channels within the $2.4 \mathrm{GHz}$ spectrum, thus ensures that interference on one or several channels will not occur. The other techniques are Direct Sequence Spread Spectrum (DSSS) coding and Time Synchronized Mesh Protocol (TSMP). The purpose of DSSS coding is to avoid network jamming, channel sharing and improved SNR ratio whereas TSMP provides a time synchronisation in scheduling the information transmission within the networks, and it is likely to reduce the battery consumption and interference. These criterions seem vital to convey and adapt MSSR in RF wireless multi-hop networks environment to scavenge multiple sources including CCI.

A multi-hop network can be defined as point-to-point node between a source and destination with the presence of intermediate relay. The performance analysis, such as relay operation policy, relay selection and power allocation are discussed in [3]. In multi-hop relaying networks, a cooperative relaying technique is beneficial to overcome fading and attenuation of the transmission signal due to its propagation path condition and distance. This improves the network performance in terms of efficiency and reliability. Thus, it is suitable to apply energy constraint networks, such as RF EH network at the relay nodes. In cooperative relaying RF EH network, most of the works focused on improving the performance gain of physical layer (i.e. relay operation) and Multiple Access Control (MAC) layer (i.e. power allocation) and network layer (i.e. relay selection). For simplicity, the cooperative relaying only considers perfect channel state information (CSI) or otherwise it becomes complex.

RF wireless networks can transport information as well as power signal from source to its destination [4] and [5]. As in [6]-[8] the proposed two (2) schemes in DF model with relaying scheme, namely Time Switching Relaying (TSR) and PSR have been introduced for Simultaneous Wireless Information and Power Transfer (SWIPT). The work is an enhancement in [4] where it is realized on pointto-point system and a single source of EH. However, the works in [6]-[8] only assume a single transmitter with a single relay in cooperative network without any multiple sources assisted including CCI.

In the conventional relaying concept, CCI within the same bandwidth as transmitted signal will deteriorate the overall system performance and should be eliminated by applying interference alignment approach or by decoding the interfering signal as stated in [9] and [10]. In this work, CCI signal is treated as a new source of EH rather than a waste energy. Particularly, in [11] and [12], the authors propose a system model in DF network using time switching scheme where the constraint relay node harvests energy from both source and interference signals, and uses to decode the source signal and forwards it to the destination. The aim is to investigate the overall system performance in terms of EH ratio for different values of average SNR and signal-to-interference ratio (SIR) received at the relay node [11] and to investigate the second hop network outage performance at the destination node [12]. The results in [11] conclude that there is a desirable outcome in exchanging the values of SNR, SIR and EH ratio at the relay node. It means as SIR increases with a fixed SNR, the optimal value of EH ratio increases. This is because when the received average SNR is constant, the increase power in CCI signal can degrade the system performance, but reduces the optimal value of EH ratio effectively. Whereas, the results in [12] are obvious as compared to the previous works without CCI assistance [6]-[8] where the overall throughput is enhanced with better outage performance. The drawback of the previous works [6]-[8] is that the numerical equation does not consider multi-source as the primary signals in contrast to the work which considers multi-interference from other networks. This motivates the work to implement a multi-source as primary signals and CCI as secondary signals as part of harvested power at the relay node.

The objectives of the paper are to develop relaying protocol for TSR and PSR in numerical form by applying multi-source with interference signals assisted and to validate the proposed technique in relation to overall system throughput against the EH ratio. The paper is organized in such that the overview and introduction of the work is discussed in part I while the system model is presented in part II. In part III is the development of numerical equations of MSSR in relation to TSR and PSR schemes and the results are discussed in part IV. 


\section{SYSTEM MODEL}

The transmission protocol used in this simulation is decode-and-forward relaying network. The protocol describes and analyzes the system throughput in the presence of energy harvesting constraint at the relay. It is assumed that the relay is an energy constraint node and capable of energy harvesting from the RF signal. The signal data is being transmitted from the source and processed at the relay prior to forwarding it to destination. The computed mathematical results are based on ergodic capacity, fraction of split power and average SNR/SIR.

As shown in system model for MSSR in Figure 1, the energy harvesting at the relay node is a combination of multiple sources' power and co-channel interferers' power in different wireless network segmentation. As of MSSR from multiple sources, $S_{1}, \ldots, S_{M}$ to relay $\mathrm{R}$, the distance represents as $d_{S 1}, \ldots, d_{S M}$ whereas from multiple interferers, $\beta_{j}, \ldots, \beta_{N}$ to relay $\mathrm{R}$, the distance represents as $d_{\beta 1}, \ldots, d_{\beta N}$ whereas the received channel for multiple sources represent as $y_{S 1}, \ldots, y_{S M}$ and for interferers represent as $y_{\beta 1}, \ldots, y_{\beta N}$. Whereby from relay $\mathrm{R}$ to destination $\mathrm{D}$, the distance represents as $d_{2}$ and received channel represents as $y_{D}$.

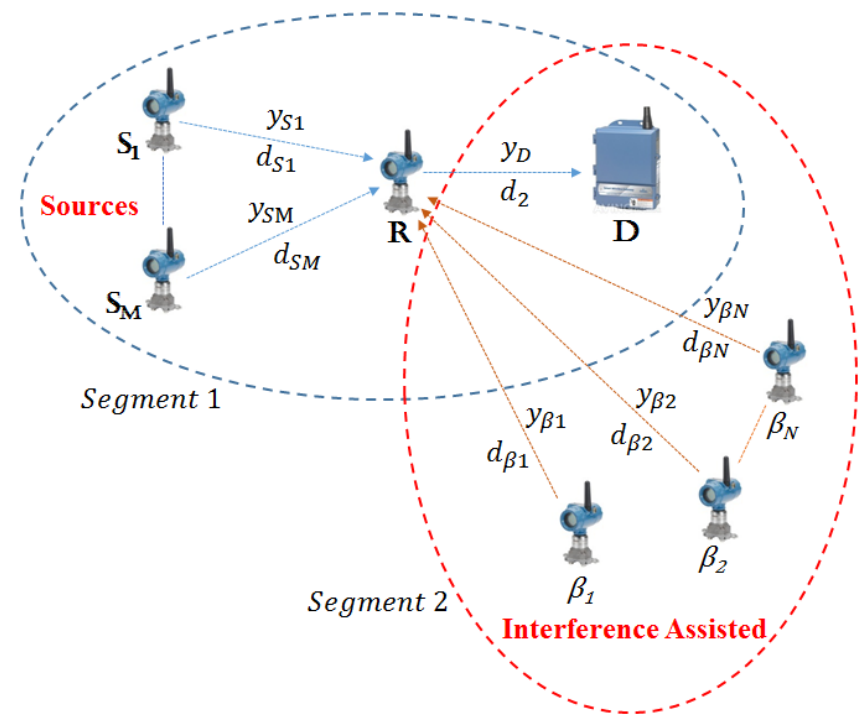

Figure 1. Schematic Diagram for 3-Node with Multiple Sources and Single Relay with Co-Channel Interference (CCI) in Mesh Topology Network

The data rate equation at $\mathrm{R}$ and $\mathrm{D}$ are shown in equations (1) where $X$ and $Y$ are two random variables of point-to-point discrete memoryless channel (DMC) which corresponds to the input and output sequences for the DF model. Discrete channels for each of the nodes are symbolized as $X_{S 1}, \ldots, X_{S M}$, $X_{\beta 1}, \ldots, X_{\beta N}$ for source nodes, $X_{R}$ and $Y_{R}$ for relay node and $Y_{D}$ for destination node. As for the signal transmission for cooperative relaying network theory, the first time slot, T1 represents signal transmission from $S_{1}, \ldots, S_{M}$ and $\beta_{j}, \ldots, \beta_{N}$ to R, while the second time slot, T2 represents signal transmission from R to D. The channel gain or Rayleigh fading channel for $S_{1}, \ldots, S_{M}$ and $\beta_{j}, \ldots, \beta_{N}$ to $\mathrm{R}$ denotes as $h_{i}$ and $\beta_{j}$ respectively while from $\mathrm{R}$ to $\mathrm{D}$ denotes as $g$. The overall addictive white Gaussian noise (AWGN) is added at both relay and destination nodes, and it represents as $n_{R}$ and $n_{D}$ respectively. $M$ denotes the no. of sources and $N$ denotes the no. of interferers.

The achievable DF rate equation which is derived from [13] for multi-source single-relay with interference assisted can be defined as:

$$
\left\{\begin{array}{l}
\mathrm{R}_{1} \leq \mathrm{I}\left(\mathrm{X}_{\mathrm{S} 1}, \ldots, \mathrm{X}_{\mathrm{SM}}, \mathrm{X}_{\beta 1}, \ldots, \mathrm{X}_{\beta \mathrm{N}} ; \mathrm{Y}_{\mathrm{R}} \mid \mathrm{X}_{\mathrm{R}}\right) \text { for Relay } \\
\mathrm{R}_{2} \leq \mathrm{I}\left(\mathrm{X}_{\mathrm{S} 1}, \ldots, \mathrm{X}_{\mathrm{SM}}, \mathrm{X}_{\beta 1}, \mathrm{X}_{\mathrm{R}} ; \mathrm{Y}_{\mathrm{D}}\right) \text { for Destination }
\end{array}\right.
$$

The achievable rate $\mathrm{R} \_1$ at the relay $\mathrm{R}$ and $\mathrm{R} \_2$ at the destination $\mathrm{D}$ can be written as: 


$$
\left\{\begin{array}{c}
R_{1} \leq \log \left(1+\frac{P_{1}}{n}\right) \\
R_{2} \leq \log \left(1+\frac{P_{2}}{n}\right) \\
R_{1}+R_{2} \leq \log \left(1+\frac{P_{1}+P_{2}}{n}\right)
\end{array}\right.
$$

where $P_{1}$ and $P_{2}$ are the signal power for data rate $R_{1}$ and $R_{2}$ accordingly and $n$ is AWGN. The achievable rate, $R_{1}$ and $R_{2}$ are expected to be nearer to that of the optimal Shannon Hartley theorem. Time slot representation of the received signal at both relay and destination nodes are denoted as:

Time slot no. 1, T1:

$$
y_{R}=\sum_{i=1}^{M} \sqrt{P_{i}} h_{i} x_{i}+\sum_{j=1}^{N} \sqrt{P_{j}} \beta_{j} x_{j}+n_{R}
$$

Time slot no. 2, T2:

$$
y_{D}=\sqrt{P_{R}} g \bar{x}+n_{D}
$$

where $y_{R}=$ the received signal at the relay, $y_{D}=$ the received signal at the destination, $h_{i}=$ channel gain from source $i$ to relay, $g=$ channel gain from relay to destination, $\beta_{j}=$ channel gain from interferer $j$ to relay, $x_{i}=$ information symbol from source $i$ to relay, $x_{j}=$ information symbol from interferer $j$ to relay, $\bar{x}=$ decoded version of the signal $x, P_{i}=$ transmitted power from source $i, P_{j}=$ transmitted power from interferer $j, P_{R}=$ transmitted power from relay, $M=$ the no. of sources, $N=$ the no. of interferers, $n_{R}=$ noise at the relay, $n_{D}=$ noise at the destination.

The transmission block structure in TSR scheme for energy harvesting and information processing at the relay is presented in Figure 2. In TSR scheme, energy harvesting power from S to R and R to D is given in (5). From the energy harvesting power, the received signal at the destination node can be derived accordingly. The same is adopted for the PSR scheme where the only difference is the energy harvesting equation from $\mathrm{S}$ to $\mathrm{R}$ and $\mathrm{R}$ to $\mathrm{D}$.

\subsection{Time Switching for MSSR}

\subsection{1. $\quad S \Rightarrow R$ Combination of Energy Harvesting and Information Transmission with Multiple Sources}

In this model, the energy constraint at relay node harvests energy from both source and interference signals, and uses the harvested energy to decode the source signal and forward it to the destination. As depicted in Figure 2, $T$ is the total block time in TSR scheme in which the information signal is transmitted from the source node to the destination node. The $\alpha \in(0,1)$ denotes the fraction of block time in which the relay harvests energy from the source and interferer signals, while the remaining block time represents as $(1-$ $\alpha) T$ is used for the information transmission. In this manner, half of the $(1-\alpha) T$ which is $(1-\alpha) T / 2$ is used for the source to relay information transmission, and the remaining half is used for the relay to destination information transmission. It is assumed that the relay would consume all the harvested energy prior to forwarding the source signal to its destination. The fraction of time $\alpha$ controls the achievable throughput at the destination node. The only difference in this MSSR as compared to a single source is the availability of energy harvesting sources which are the sum of source and interferers. The details presentation of the received signal at the relay node and destination node is analyzed in below section.

\section{T}

\begin{tabular}{|c|c|c|}
\hline $\begin{array}{c}S ; \beta \\
\text { EH at Relay }\end{array}$ & $\begin{array}{c}S \Rightarrow \mathrm{R} \\
\text { Information Transmission }\end{array}$ & $\begin{array}{c}\mathrm{R} \Rightarrow \mathrm{D} \\
\text { Information Transmission }\end{array}$ \\
\hline$\alpha T$ & \multicolumn{2}{|}{$(1-\alpha) T / 2$} \\
\hline
\end{tabular}

Figure 2. Transmission Block Structure in TSR Scheme for EH and Information Transmission

The received signal, $i$ at the relay node, $y_{R}(k)$ can be derived as: 


$$
\begin{aligned}
& y_{R}(k)=\sum_{i=1}^{M} \frac{1}{\sqrt{d_{i}^{m}}} \sqrt{P_{i}} h_{i} s_{i}(k)+ \\
& \sum_{j=1}^{N} \frac{1}{\sqrt{d_{j}^{m}}} \sqrt{P_{j}} \beta_{j} s_{j}(k)+n_{R}(k)
\end{aligned}
$$

where $k=1,2, \ldots$,integer denotes symbol index, $d_{i}=$ distance from source $i$ to relay, $d_{j}=$ distance from interferer $j$ to relay, $h_{i}=$ channel gain from source $i$ to relay, $P_{i}=$ transmitted power from source $i, \beta_{j}=$ channel gain from interferer $j$ to relay, $P_{j}=\operatorname{transmitted~power~from~interferer~} j, m=$ path loss exponent, $M=$ the no. of sources, $N=$ the no. of interferers, $s_{i}(k)=k_{\text {th }}$ normalized information symbol from the source $i$, i.e. $\mathrm{E}\left\{\left|s_{i}(k)\right|^{2}\right\}=1, s_{j}(k)=k_{\text {th }}$ normalized information symbol from the interferer $j$, i.e. $\mathrm{E}\left\{\left|s_{j}(k)\right|^{2}\right\}=1$, $n_{R}(k)=$ baseband addictive white Gaussian noise (AWGN) at the relay.

The energy harvesting during $\alpha T$ time can be written as:

$$
E_{h}^{T S}=\eta\left(\sum_{i=1}^{M} \frac{1}{d_{i}^{m}} P_{i}\left|h_{i}\right|^{2}+\sum_{j=1}^{N} \frac{1}{d_{j}^{m}} P_{j}\left|\beta_{j}\right|^{2}\right) \alpha T
$$

where $\eta=$ energy conversion efficiency is $0<\eta<1$ which depends on energy harvesting efficiency and the rectification process.

\subsection{2. $\quad \mathbf{R} \Rightarrow \mathbf{D}$ Information Transmission}

The decoded source signal at the relay node is forwarded to the destination node with power $P_{R}^{T S}$ as described in earlier section. The power use for the information transmission is the available harvested energy power during energy harvesting period. The received signal at the destination node, $y_{D}^{T S^{M S S R}}(k)$ is given as:

$$
y_{D}^{T S^{M S S R}}(k)=\frac{1}{\sqrt{d_{R D}^{m}}} \sqrt{P_{R}^{T S}} g \cdot \bar{s}(k)+n_{D}(k)
$$

where $\quad d_{R D}=$ distance from relay to destination, $g=$ channel gain from relay to destination, $P_{R}=$ transmitted power from relay, $\bar{s}(k)=$ decoded version of the signal $s(k), n_{D}(k)=$ baseband addictive white Gaussian noise (AWGN) at the destination.

The transmitted power from the relay node for $(1-\alpha) T / 2$ time in relation to the harvested energy $E_{h}^{T S}$ can be written as:

Substitutes (6) into (8):

$$
P_{R}^{T S}=\frac{E_{h}^{T S}}{(1-\alpha) T / 2}
$$

$$
P_{R}^{T S}=\frac{\eta\left(\sum_{i=1}^{M} \frac{1}{d_{i}^{m}} P_{i}\left|h_{i}\right|^{2}+\sum_{j=1}^{N} \frac{1}{d_{j}^{m}} P_{j}\left|\beta_{j}\right|^{2}\right) \alpha T}{(1-\alpha) T / 2}=\frac{2 \eta \alpha\left(\sum_{i=1}^{M} \frac{1}{d_{i}^{m}} P_{i}\left|h_{i}\right|^{2}+\sum_{j=1}^{N} \frac{1}{d_{j}^{m}} P_{j}\left|\beta_{j}\right|^{2}\right)}{(1-\alpha)}
$$

Substitutes (9) into (7) will yield the received signal at the destination node:

$$
y_{D}^{T S^{M S S R}}(k)=\sqrt{\frac{2 \eta \alpha\left(\sum_{i=1}^{M} \frac{1}{d_{i}^{m}} P_{i}\left|h_{i}\right|^{2}+\sum_{j=1}^{N} \frac{1}{d_{j}^{m}} P_{j}\left|\beta_{j}\right|^{2}\right)}{d_{R D}^{m}(1-\alpha)}} g \bar{s}(k)+n_{D}(k)
$$

\subsubsection{Throughput Analysis}

In considering (5), the SNR/SIR at the relay node, $\gamma_{R}^{T S^{M S S R}}$ can be derived as:

$$
\gamma_{R}^{T S^{M S S R}}=\sum_{i=1}^{M} \sum_{j=1}^{N} \frac{P_{i}\left|h_{j}\right|^{2} d_{j}^{m}}{P_{j}\left|\beta_{j}\right|^{2} d_{i}^{m}+\sigma_{n_{R}^{T S}}^{2}}
$$

where $\sigma_{n_{R}^{T S}}^{2} \triangleq \sigma_{n_{R}}^{2}$ is the variance of the overall AWGN at the relay node, i.e. $n_{R}^{T S} \triangleq n_{R}(k)$

Using (10), the SNR/SIR at the destination node, $\gamma_{D}^{T S^{M S S R}}$ can be derived as:

$$
\gamma_{D}^{T S^{M S S R}}=\sum_{i=1}^{M} \sum_{j=1}^{N} \frac{2 \eta \alpha\left(P_{i} P_{j}\left|h_{i}\right|^{2}\left|\beta_{j}\right|^{2}\right)|g|^{2}}{d_{i}^{m} d_{j}^{m} d_{R D}^{m} \sigma_{n_{D}}^{2}(1-\alpha)}
$$

where $\sigma_{n_{D}}^{2}$ is the variance of the overall AWGN at the destination node. 
For the achievable throughput of the ergodic capacity for source to relay link, $\mathrm{C}_{R}{ }^{T S}$ and for relay to destination link, $\mathrm{C}_{D}{ }^{T S}$ is evaluated and determined using the received SNR/SIR for both link respectively. Thus, the ergodic capacity of source to relay link can be written as:

$$
\mathrm{C}_{R}^{T S}=\mathrm{E}_{\mathrm{h}, \beta}\left\{\log _{2}\left(1+\gamma_{R}^{T S^{M S S R}}\right)\right\}
$$

whereas, the ergodic capacity of relay to destination link is given by:

$$
\mathrm{C}_{D}^{T S}=\mathrm{E}_{\mathrm{h}, \beta, \mathrm{g}}\left\{\log _{2}\left(1+\gamma_{D}^{T S^{M S S R}}\right)\right\}
$$

\subsection{Power Splitting for MSSR}

\subsection{1. $\quad S \Rightarrow R$ Combination of Energy Harvesting and Information Transmission with Multiple Sources}

As depicted in Figure 3, $\mathrm{T}$ is the total block time in PSR scheme in which the received power signal from the source and interferer, $\mathrm{P}$ is split at the relay node for the energy harvesting and information signal prior to sending the decoded source signal to the destination node. The $\rho \in(0,1)$ denotes the fraction of power in which the relay harvests energy from the source and interferer signals with the fraction of $\rho \_(i, j) P$, while the remaining fraction of power $\left(1-\rho_{-}(i, j)\right) P$ is used for the information transmission. In this manner, the first half of the block time $\mathrm{T} / 2$ is used for the source to relay information transmission, and the remaining half $\mathrm{T} / 2$ is used for the relay to destination information transmission. The fraction of power $\rho$ controls the achievable throughput at the destination node. The details presentation of the received signal at the relay node and destination node is analyzed in below section

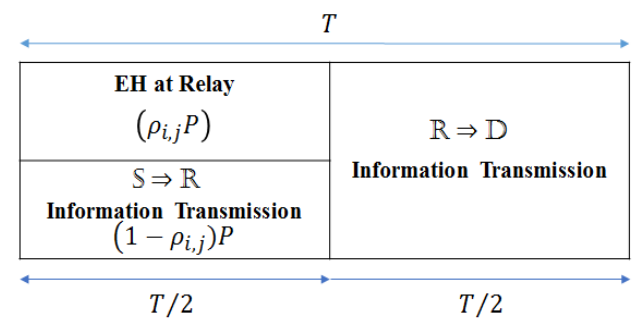

Figure 3. Transmission Block Structure in PSR Scheme for EH and Information Transmission

In PSR scheme, power is split into 2 proportions as illustrated in Figure 2, where the first portion of the received signal, $\sum_{i=1}^{M} \sum_{j=1}^{N} \sqrt{\rho_{i}} \sqrt{\rho_{j}} y_{R}(k)$ will be sent to $\mathrm{EH}$ receiver and the second portion of the received signal, $\sum_{i=1}^{M} \sum_{j=1}^{N} \sqrt{1-\rho_{i}} \sqrt{1-\rho_{j}} y_{R}(k)$ will be sent to information receiver.

$$
\begin{aligned}
& \underbrace{\sum_{j=1}^{M} \sqrt{\rho_{i}} \sqrt{\rho_{j}} y_{R}(k): \underbrace{\sum_{i=1}^{M} \sum_{j}^{N} \sqrt{1-\rho_{i}} \sqrt{1-\rho_{j}} y_{R}(k)}_{i=1}}_{i=1} \\
& \mathrm{EH} \quad \text { Information }
\end{aligned}
$$

The received signal at the relay node, $y_{R}(k)$ is defined as:

$$
\begin{gathered}
\sqrt{\rho_{i}} \sqrt{\rho_{j}} y_{R}(k)=\sum_{i=1}^{M} \frac{1}{\sqrt{d_{i}^{m}}} \sqrt{\rho_{i} P_{i}} h_{i} s_{i}(k)+ \\
\sum_{j=1}^{N} \frac{1}{\sqrt{d_{j}^{m}}} \sqrt{\rho_{j} P_{j}} \beta_{j} s_{j}(k)+\sqrt{\rho_{i}} \sqrt{\rho_{j}} n_{R}(k)
\end{gathered}
$$

where $k=1,2, \ldots$, integer denotes symbol index, $d_{i}=$ distance from source $i$ to relay, $d_{j}=$ distance from interferer $j$ to relay, $h_{i}=$ channel gain from source $i$ to relay, $P_{i}=$ transmitted power from source $i, \beta_{j}=$ channel gain from interferer $j$ to relay, $P_{j}=$ transmitted power from interferer $j, \mathrm{M}=$ the no. of sources, $\mathrm{N}=$ the no. of interferers, $m=$ path loss exponent, $s_{i}(k)=k_{\text {th }}$ normalized information symbol from the source $i$, 
i.e. $\mathrm{E}\left\{\left|s_{i}(k)\right|^{2}\right\}=1, s_{j}(k)=k_{\text {th }}$ normalized information symbol from the interferer $j$, i.e. $\mathrm{E}\left\{\left|s_{j}(k)\right|^{2}\right\}=1$, $n_{R}(k)=$ baseband addictive white Gaussian noise (AWGN) at the relay.

The energy harvesting, $E_{h}^{P S}$ during $T / 2$ time can be written as:

$$
E_{h}^{P S}=\eta\left(\sum_{i=1}^{M} \frac{1}{d_{i}^{m}} \rho_{i} P_{i}\left|h_{i}\right|^{2}+\sum_{j=1}^{N} \frac{1}{d_{j}^{m}} \rho_{j} P_{j}\left|\beta_{j}\right|^{2}\right) T / 2
$$

The baseband signal at the input of information receiver after the power split is:

$$
\begin{array}{r}
\sqrt{1-\rho_{i}} \sqrt{1-\rho_{j}} y_{R}(k)=\sum_{i=1}^{M} \frac{1}{\sqrt{d_{i}^{m}}} \sqrt{1-\rho_{i} P_{i}} h_{i} s_{i}(k) \\
+\sum_{j=1}^{N} \frac{1}{\sqrt{d_{j}^{m}}} \sqrt{1-\rho_{j} P_{j}} \beta_{j} s_{j}(k)+\sqrt{1-\rho_{i}} \sqrt{1-\rho_{j}} n_{R}(k)
\end{array}
$$

\subsection{2. $\quad \mathrm{R} \Rightarrow \mathrm{D}$ Information Transmission}

The decoded source signal at the relay node is forwarded to the destination node with power $P_{R}^{P S}$ as described in earlier section. The power use for the information transmission is the available harvested energy power during energy harvesting period. The received signal at the destination node, $y_{D}^{P S^{M S S R}}(k)$ is given as:

$$
y_{D}^{P S^{M S S R}}(k)=\frac{1}{\sqrt{d_{R D}^{m}}} \sqrt{P_{R}^{P S}} g \cdot \bar{s}(k)+n_{D}(k)
$$
written as:

The transmitted power from relay node for $T / 2$ time in relation to the harvested energy $E_{h}^{P S}$ can be

Substitutes (16) into (19):

$$
P_{R}^{P S}=\frac{E_{h}^{P S}}{T / 2}
$$

$$
P_{R}^{P S}=\frac{\eta\left(\sum_{i=1}^{M} \frac{1}{d_{i}^{m}} \rho_{i} P_{i}\left|h_{i}\right|^{2}+\sum_{j=1}^{N} \frac{1}{d_{j}^{m}} \rho_{j} P_{j}\left|\beta_{j}\right|^{2}\right) T / 2}{T / 2}=\eta\left(\sum_{i=1}^{M} \frac{1}{d_{i}^{m}} \rho_{i} P_{i}\left|h_{i}\right|^{2}+\sum_{j=1}^{N} \frac{1}{d_{j}^{m}} \rho_{j} P_{j}\left|\beta_{j}\right|^{2}\right)
$$

Substitutes (20) into (18) will yield the received signal at the destination node:

$$
y_{D}^{P S^{M S S R}}(k)=\sqrt{\frac{\eta\left(\sum_{i=1}^{M} \frac{1}{\left.d_{i}^{m} \rho_{i} P_{i}\left|h_{i}\right|^{2}+\sum_{j=1}^{N} \frac{1}{d_{j}^{m} \rho_{j} P_{j}}\left|\beta_{j}\right|^{2}\right)}\right.}{d_{R D}^{m}}} g \bar{S}(k)+n_{D}(k)
$$

\subsubsection{Throughput Analysis}

In considering (17), the SNR/SIR at the relay node, $\gamma_{R}^{P S^{M S S R}}$ can be derived as:

$$
\gamma_{R}^{P S^{M S S R}}=\sum_{i=1}^{M} \sum_{j=1}^{N} \frac{P_{i}\left|h_{j}\right|^{2}\left(1-\rho_{i}\right) d_{j}^{m}}{P_{j}\left|\beta_{j}\right|^{2}\left(1-\rho_{j}\right) d_{i}^{m}+\sigma_{n_{R}^{P S}}^{2}}
$$

where $\sigma_{n_{R}^{P S}}^{2} \triangleq\left(1-\rho_{i}\right)\left(1-\rho_{j}\right) \sigma_{n_{R}}^{2}$ is the variance of the overall AWGN at the relay node, i.e. $n_{R}^{P S} \triangleq n_{R}(k)$

Using (21), the SNR/SIR at the destination node, $\gamma_{D}^{P S^{M S S R}}$ can be derived as:

$$
\gamma_{D}^{P S^{M S S R}}=\sum_{i=1}^{M} \sum_{j=1}^{N} \frac{\eta P_{i} P_{j}\left|h_{i}\right|^{2}\left|\beta_{j}\right|^{2}|g|^{2} \rho_{i} \rho_{j}}{d_{i}^{m} d_{j}^{m} d_{R D}^{m} \sigma_{n_{D}}^{2}}
$$

where $\sigma_{n_{D}}^{2}$ is the variance of the overall AWGN at the destination node.

For the achievable throughput of the ergodic capacity for source to relay link, $\mathrm{C}_{R}{ }^{P S}$ and for relay to destination link, $\mathrm{C}_{D}{ }^{P S}$ is evaluated and determined using the received SNR/SIR for both link respectively. Thus, the ergodic capacity of source to relay link can be written as:

$$
\mathrm{C}_{R}{ }^{P S}=\mathrm{E}_{h}\left\{\log _{2}\left(1+\gamma_{R}^{P S^{M S S R}}\right)\right\}
$$

whereas, the ergodic capacity of relay to destination link is given by:

$$
\mathrm{C}_{D}{ }^{P S}=\mathrm{E}_{h, g}\left\{\log _{2}\left(1+\gamma_{D}^{P S^{M S S R}}\right)\right\}
$$

\section{NUMERICAL ANALYSIS}

This section discusses numerical results and simulation analysis for MSSR. All related parameters and values are selected based on MSSR equations. Unless stated otherwise, the number of source $M$ is set to 2 , while the number of interferer, $N$ is set to 2 . Integers $i$ and $j$ are referring to $M$ and $N$ accordingly. The average SNR and SIR values are set to $20 \mathrm{~dB}$ respectively. The energy harvesting efficiency, $\eta=0.7$, distance 
from sources $i$ to relay, $d_{i}=1$, distance from interferers $j$ to relay, $d_{j}=1$, distance from relay to destination, $d_{R D}=1$, transmitted power from sources, $P_{i}=1$ watt, transmitted power from interferers, $P_{j}=1$ watt, noise power at relay, $\sigma_{n_{R}}^{2}=0.1$ watt, noise power at destination, $\sigma_{n_{D}}^{2}=0.1$ watt, pathloss exponent, $m=2.7$ and the target rate, $R=1$.

The trending plots for ideal receiver using SSSR and MSSR can be seen in Figure 4 and 5. The system throughput versus the energy harvesting ratio with respect to the fraction of block time, $\alpha$ for TSR scheme is illustrated in Figure 4. In this case, the SSSR model is matching perfectly with ideal receiver [8]. The concave feature of the curves from the plots explains the signal transmission from relay to destination in the second time slot. As $\alpha$ increases, the system throughput increases until it reaches its optimal value, then it starts to decrease from maximum to zero. During this period, the capacity is enhanced due to the increase of energy harvesting and the system uses all the available energy to transmit information effectively to its destination. When the EH ratio reaches its optimal value, the throughput starts reducing as more EH energy is harnessed rather than the information is decoded for the information transmission at this time fraction. From the plot, the optimal value of $\alpha$ for the peak throughput in MSSR is at 0.2 as compared to 0.3 in ideal receiver, which reduces significantly the value of $\mathrm{EH}$ ratio by $10 \%$.

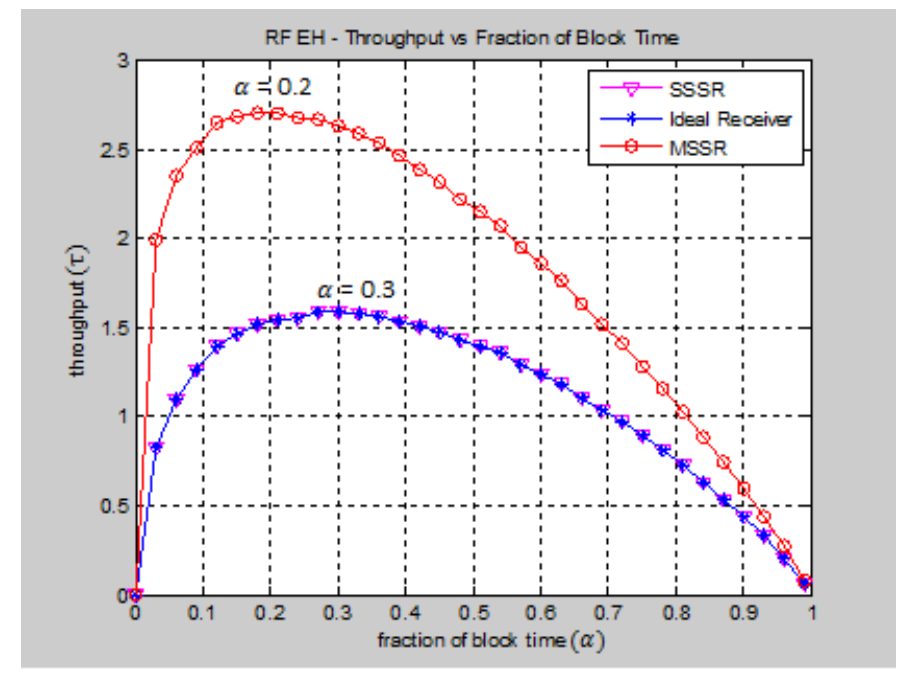

Figure 4. Throughput vs EH Ratio at Destination Node with Respect to Fraction of Block Time, $\alpha$ for TSR Scheme

In Figure 5, the system throughput versus the energy harvesting ratio with respect to the fraction of power split, $\rho$ for PSR scheme is simulated. In this case, the SSSR model is matching perfectly with ideal receiver [8]. As $\rho$ increases, the system throughput increases until it reaches its optimal value which is 0.7 , then it starts to decrease from maximum to zero. The EH ratio range between 0 to 0.7 indicates that the time slot is used further in signal decoding rather than energy harvesting. As compared to the EH ratio range between 0.7 to 1 , the time slot is used for energy harvesting rather than signal decoding. As we can see from both curves, the trend is almost identical. This is due to continuous operation of power splitting and information decoding in both first and second half of the power fractions which yields more energy being harvested and more information is decoded simultaneously during this period. From the plot, the optimal value of $\rho$ for the peak throughput in MSSR is at 0.7 as compared to 0.5 in ideal receiver. 


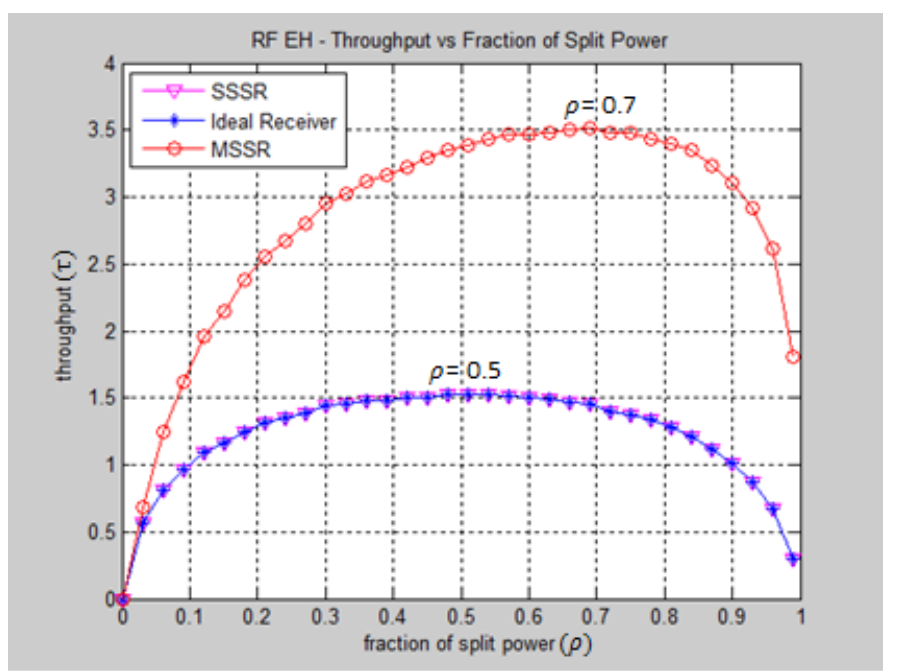

Figure 5. Throughput vs EH Ratio at Destination Node with Respect to Fraction of Split Power, $\rho$ for PSR Scheme

In the next simulation, to illustrate the benefit of MSSR with multiple sources is through cumulative distribution function (CDF). Figure 6 shows the variation of probability of CDF against system throughput. From the curves, it can be easily obtained whichever outage percentage acquired from the system. For example in Figure 6, the $20 \%$ capacity outage for the MSSR is equal to 2.6 bits/transmission as compared to a much lower of 1.5 bits/transmission for SSSR.

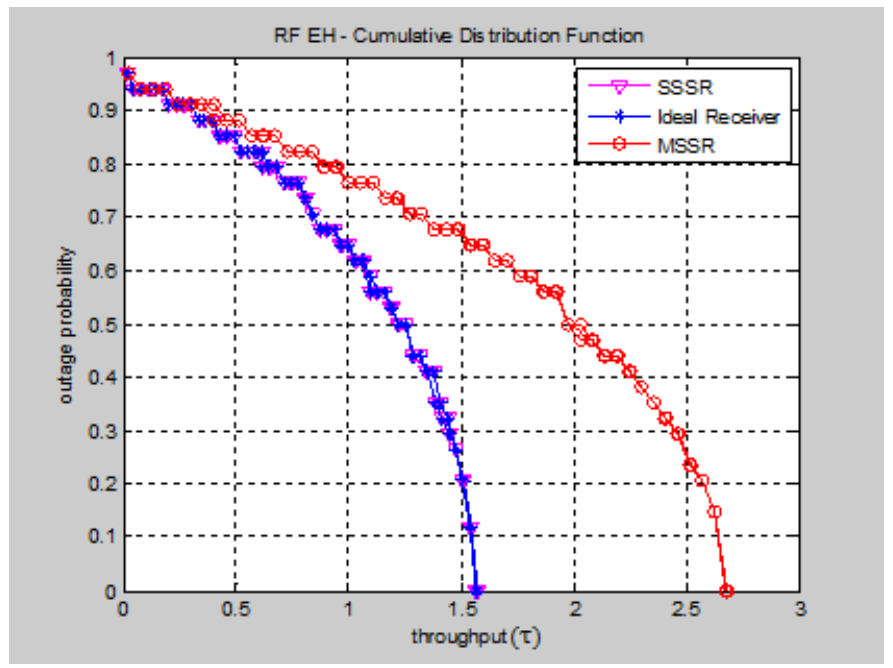

Figure 6. Variation of Probability of CDF Against Throughput with Respect to EH Ratio

Figure 7 shows the variation of probability of CDF against the selected rate. By observing at the CDF curves, it can be figured out that the outage probability decreases quite significantly for the MSSR. For example, for a selected rate of $\mathrm{R}=1.5$ bits/transmission, the outage probability for SSSR is approximately $70 \%$, while it is significantly much smaller for the MSSR which is $35 \%$. 


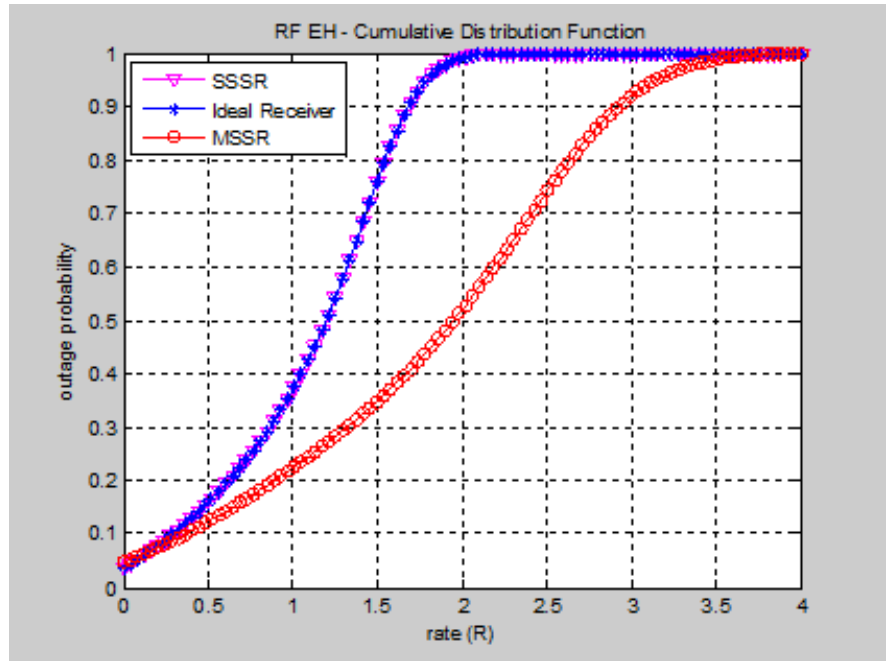

Figure 7. Variation of Probability of CDF against Selected Rate with Respect to EH Ratio

\section{CONCLUSION}

In this paper, the MSSR scheme in decode-and-forward model with multiple sources including CCI signal is proposed where the multiple sources and CCI are harvested at the constraint relay node and used the harvested energy to decode-and-forward the information signal from source to destination. Both TSR and PSR schemes have been adopted in the mathematical expression to derive the instantaneous capacity in terms of SNR/SIR. TSR scheme is adopted where the receiver is switching over stipulated time in between information transmission and energy harvesting processes, whereas PSR scheme is used when the receiver splits between information transmission and energy harvesting overtime. The optimal values of EH ratio for both TSR and PSR schemes are obtained with respect to the overall achievable throughput. It was shown in simulation that multiple sources including CCI signal has significant effect on energy harvesting in terms of harvested energy loading and consumption at the constraint node. In other word it can provide alternative energy for the relay in lieu of conventional battery.

In TSR scheme, multiple sources have substantially reduced the optimal value of EH ratio and increased the overall system throughput as compared to ideal receiver, while in PSR scheme, due to the nature of simultaneous and continuous operation for power splitting processes, the optimal value of EH ratio is decreased as compared to the ideal receiver but a trade-off in overall system throughput which is way better than the latter. As mentioned in the overview of the nature of wireless sensor coexistence with other wireless technologies, the interference cancellation techniques under lower noise variance can be utilized in order to improve overall system performance. Future work under investigation is the proposal of multiple relaying with interference assisted in energy harvesting approach to decode and forward the information signal from source to destination. The proposal will provide theoretical insights into the effect of overall system performance with various system parameters of information processing and energy harvesting using decodeand-forward model.

\section{REFERENCES}

[1] J. A. Paradiso and T. Starner. Energy Scavenging for Mobile and Wireless Electronics. IEEE Pervasive Comput. 2005; 4: 18-27.

[2] Emerson Process Management. 00805-0100-1039. The Engineer's Guide to Industrial Wireless Management. Minnesota USA. Emerson Process Management. 2014.

[3] Lu, Xiao, et al. Wireless networks with RF energy harvesting: A Contemporary Survey. IEEE Communications Surveys \& Tutorials. 2015; 17(2): 757-789.

[4] C. Zhong, H. A. Suraweera, G. Zjeng, I. Krikidis, and Z. Zhang. Wireless Information and Power Transfer with Full Duplex Relaying. IEEE Transactions on Communications. 2014; 62(2): 3447-3461.

[5] X. Zhou, R. Zhang, and C. K. Ho. Wireless Information and Power Transfer: Architecture Design and Rate-Energy Tradeoff. IEEE Transactions on Communications. 2013; 61(11): 4754-4767.

[6] P. Mangayarkarasi, J. Raja, and S. Jayashri. An Efficient Energy Harvesting Scheme to Maximize the Throughput of the Wireless Relay Network with TSR and PSR Protocol. International Journal of Electronics and Communications. 2015; 69(5): 841-850.

[7] A. A. Nasir, X. Zhou, S. Durrani, and R. A. Kennedy. Relaying Protocols for Wireless Energy Harvesting and Information Processing. IEEE Transactions on Wireless Communications. 2013; 12(7): 3622-3636. 
[8] A. A. Nasir, X. Zhou, S. Durrani, and R. A. Kennedy. Throughput and Ergodic Capacity of Wireless Energy Harvesting Based DF Relaying Network. in Proceedings of the IEEE International Conference on Communications (ICC'14), IEEE, Sydney, Australia. 2014; 4066-4071.

[9] M. Xia and S. Aissa. Cooperative AF Relaying in Spectrum-Sharing Systems: Performance Analysis Under Average Interference Power Constraints and Nakagami-m Fading. IEEE Trans. Commun. June 2012; 60(6):1523-1533.

[10] Y. Gu, S. Ikki and S. Aissa. Opportunistic Cooperative Communication in the Presence of Co-Channel Interferences and Outdated Channel Information. IEEE Commun. lett. Oct. 2013; 17(10): 1948-1951.

[11] Y. Gu and S. Aïssa. Interference Aided Energy Harvesting in Decode-and-Forward Relaying Systems. IEEE International Conference on Communications (ICC). Sydney, NSW. 2014; 5378-5382.

[12] S. S. Kalamkar and A. Banerjee. Interference-Assisted Wireless Energy Harvesting in Cognitive Relay Network with Multiple Primary Transceivers. IEEE Global Communications Conference (GLOBECOM). San Diego, CA. 2015; $1-6$.

[13] Alexander M. Wyglinski, M. Nekovee, and Y. Thomas Hou. Cognitive Radio Communications and Networks. Academic Press, Oxford. 2010; 264-276.

[14] Ahmad LP, Hooper A. The Lower Switching Losses Method of Space Vector Modulation. CN103045489 (Patent). 2007.

[15] Zainudin, M. S., Rahim, M. K. A., Samsuri, N. A., \& Majid, H. A. (2017). RF Energy Harvesting Study Using Various Metamaterial Patch Structure. International Journal of Electrical and Computer Engineering (IJECE), 7(3), 1574-1579. 